\section{(2) OPEN ACCESS}

\title{
Central catheter-associated deep vein thrombosis in cancer: clinical course, prophylaxis, treatment
}

\author{
Arnaldo Marin, ${ }^{1}$ Leonardo Bull, ${ }^{2}$ Matthew Kinzie $(1),{ }^{3}$ Max Andresen ${ }^{4}$
}

'Department of Basic and Clinical Oncology, University of Chile Faculty of Medicine, Santiago, 8380453, Chile ${ }^{2}$ School of Medicine, University of Chile, Santiago, 8380453, Chile

${ }^{3}$ Neurosciences, University of Texas Health, Houston, Texas, USA

${ }^{4}$ Intensive Care Department, Facultad de Medicina . Pontificia Universidad Catolica de Chile, Santiago, 8330077, Chile

\section{Correspondence to}

Dr Max Andresen, Intensive Care Department, Facultad de Medicina .Pontificia Universidad Catolica de Chile, Santiago, Chile; andresen@med.puc.cl

Received 15 November 2019 Accepted 7 April 2021

Published Online First

19 August 2021

\begin{abstract}
The adequate handling of central venous catheters is a key element in the management of patients with cancer. Catheter-associated deep vein thrombosis is frequently observed in patients with malignant diseases; however, despite being a common complication among these patients, objective information concerning its epidemiology, clinical course, prophylaxis and treatment strategies is very limited. The reported incidence of catheter-related thrombosis (CRT) is highly variable, depending on symptomatic events, or if patients are screened for asymptomatic thrombosis. Several factors have been identified as potential predisposing factors for CRT, both technical and pathological aspects. The anticoagulant of choice is still unclear; while low-molecular-weight heparin is most commonly used, recent studies assessing the role of direct oral anticoagulants in the treatment of CRT show promise as an alternative, but the evidence remains insufficient and the decision must be made on a case-by-case basis.
\end{abstract}

\section{THROMBOSIS AND CANCER}

Upper extremity deep vein thrombosis (UEDVT) is a common complication frequently observed in patients with malignant diseases using central venous catheters (CVCs), which are currently recognised as the main predisposing factor for secondary UEDVT. ${ }^{1}$ Despite its frequency among these patients, objective information concerning its epidemiology, clinical course, prophylaxis and treatment strategies is very limited.

Recent infection is also a factor independently associated with a 1.7-fold increased venous thromboembolism (VTE) risk, possibly due to a procoagulant state induced by the inflammatory response to infection. Additionally, infection is a VTE risk factor for hospitalised patients with cancer. This strong negative impact of VTE on the prognosis of

\section{Key messages}

What is already known on this topic

- DVT is common in central lines and cancer

- No standard for anticoagulation

What this study adds

- RIJ venipunctures pose lowest DVT CRT risk

- LIJ and multiple venipunctures increase DVT risk

- Heparin or direct oral anticoagulants may be used long-term

- Empirical treatment rather than central line removal and reinsertions should be considered

- Further research needed for best preventative treatment

patients with cancer has been reported on all major clinical presentations of VTE: deep vein thrombosis (DVT), whether proximal or distal, and pulmonary embolism (PE). ${ }^{2}$ Even worse, a recent study suggested that patients with cancer with superficial venous thrombosis have a poor prognosis, similar to that of patients with cancer-related DVT. Thus, the high rate of DVT-PE recurrence suggests that such patients may need longer duration of anticoagulant treatment. ${ }^{3}$

\section{CVC AND CANCER}

CVC use is a clinical practice with an ever-growing frequency worldwide. Patients with cancer typically require central venous catheterisation to keep peripheral veins from harm in the setting of chemotherapy, hematopoietic stem cell transplantation, or transfusion of blood products and components. However, CVC use is not devoid of risks and potential complications, such as infection, thrombosis and immediate complications related to the insertion procedure.

Oncological patients constitute a population at high risk of VTE. It is widely 
known that malignant neoplastic disease generates a hypercoagulable state, a consequence of prothrombotic factors resulting from the inflammatory response to the neoplasm. CVCs have been identified as an independent factor associated with an 8.5-fold increased risk of VTE in patients with cancer. ${ }^{4}$ Although multiple different devices exist, the presence of a central line heightens the risk of VTE.

\section{EPIDEMIOLOGY}

The reported incidence of CVC-related thrombotic events is highly variable and depends on whether patients were screened asymptomatically, or if only symptomatic events were reported. It is not uncommon for catheter-related thrombosis (CRT) to present without any local signs or symptoms. Therefore, if only symptomatic - neck pain or swelling-CRT is considered, then one should expect to miss some thrombotic events and the real incidence is underestimated because $5 \%-41 \%$ of patients developed such symptomatic thrombi. ${ }^{5}$ The screening method is also a relevant factor. Thus, studies using venography tend to yield higher incidence rates than those using ultrasonography.

\section{ASYMPTOMATIC EVENTS: VENOGRAPHY Prospective studies}

There are a few studies that incorporated venography for CRT screening. Such invasive techniques are seldom used for this purpose in clinical settings but give valuable insight into the real frequency of these events happening. A randomised, double-blind, placebo-controlled study assessing the efficacy and safety of low-molecular-weight heparin (LMWH) in the prevention of CVC-associated VTE reported a global incidence of $16.1 \%(14.1 \%$ in the $\mathrm{LMWH}$ group vs $18 \%$ in the placebo group, $\mathrm{p}=0.35){ }^{6}$ However, much greater rates have been reported by other studies screening with this modality. Studies using venography tend to yield higher incidence rates than those using ultrasonography. A prospective study assessing the prevalence of thrombotic complications related to CVCs in patients with cancer described a high prevalence of thrombotic events (66\%), of which only $6 \%$ developed symptoms. ${ }^{7}$

\section{ASYMPTOMATIC EVENTS: SONOGRAPHY Prospective studies}

Less invasive screening methods such as ultrasonography have been used; their results are highly heterogeneous. A prospective cohort study following a group of patients with cancer who underwent longterm CVC implantation reported an incidence of 0.10 events per 1000 catheter-days $(<1.5 \%){ }^{8}$ In contrast, another prospective study of a similar design yielded a considerably higher incidence rate of $11.7 \% .^{9}$ Another cohort study following a group of haematological malignancies evaluated if clinical thrombosis could be predicted by screening with Doppler ultrasound. The reported cumulative incidence of subclinical thrombosis was $24.8 \%$, while the incidence of symptomatic thrombosis was $12.4 \% .^{10}$

\section{SYMPTOMATIC EVENTS \\ Prospective studies}

A randomised multicentre clinical trial comparing CVC-related complications in patients with nonhaematological malignancies with peripherally inserted central catheter (PICC) lines versus patients with portchamber catheters resulted in a CVC-related thrombosis incidence of $25 \%$ in the PICC arm, in contrast, a $0 \%$ incidence in the port-chamber arm. ${ }^{11} 12$

A prospective observational study assessing the safety of CVCs in patients with cancer reported a $4.5 \%$ incidence of CVC-related thrombosis. ${ }^{12}$ A prospective observational multicentre study assessed the incidence and risk factors of CVC-related thrombotic events in patients with haematological malignancies. The result was an overall incidence rate of $12 \%{ }^{13}$

\section{Retrospective studies}

There have been several retrospective studies related to CRT in patients with cancer. A retrospective cohort analysis of a healthcare claims database that compared the reported incidence of CVC-related complications of patients with cancer with patients without cancer showed statistically significantly higher rates of thrombosis in the cancer group (1.71 vs 0.76 events per 1000 catheter-years). ${ }^{14}$ Another retrospective cohort analysis using health insurance claims, following a group of patients with breast cancer undergoing chemotherapy, assessed the incidence of complications comparing long-term CVC implantation versus temporary intravenous catheters. In the long-term CVC group, 9\% had a thrombovascular complication, while in the transient CVC group the incidence was significantly lower (4\%). ${ }^{15}$

In a retrospective study of VTE in patients with acute lymphoblastic leukaemia (ALL) and acute myeloid leukaemia (AML), an overall prevalence of $10.7 \%$ was reported. Most of the thrombotic events registered were CVC related (ALL: 83\%, AML: 77.9\%). ${ }^{16}$

A retrospective study assessing the safety and effectiveness of long-term, tunnelled, non-cuffed CVCs reported a CVC-related thrombosis incidence of $4.3 \%$, most of which happened in patients with haematological malignancies (10 cases vs 3 cases with solid tumours). ${ }^{17}$

A recent retrospective study reported an incidence of PICC-related symptomatic thrombosis of 0.15 per 1000 PICC-days. ${ }^{18}$ Older studies have described that intraluminal thrombosis requiring catheter removal can occur at a frequency of $0.6-0.81$ events per 1000 catheter-days. ${ }^{19}$ Table 1 summarises the incidence of asymptomatic and symptomatic CRT by type of catheter in patients with cancer. 
Table 1 Incidence of asymptomatic and symptomatic CRT by type of catheter in patients with cancer

\begin{tabular}{lllll}
\hline Catheter type & Study type & Thrombosis type & Incidence & Reference \\
\hline CICC & Retrospective & Symptomatic & $4.3 \%$ & 17 \\
CICC & Retrospective & Symptomatic & $22 \%$ & 19 \\
CICC & Prospective & Symptomatic & $6.5 \%$ & 26 \\
CICC & Prospective & Asymptomatic & $24.8 \%$ & 10 \\
CICC & Prospective & Symptomatic & $12.4 \%$ & 10 \\
\hline Port & Prospective & Asymptomatic & $1.25 \%$ & 8 \\
Port & Prospective & Asymptomatic & $11.7 \%$ & 9 \\
Port & Prospective & Symptomatic & $0 \%$ & 11 \\
Port & Prospective & Symptomatic & $3.4 \%$ & 50 \\
PICC & Prospective & Symptomatic & $25 \%$ & 11 \\
PICC & Prospective & Symptomatic & $12.5 \%$ & 12 \\
PICC & Prospective & Symptomatic & $9.7 \%$ & 50 \\
PICC & Retrospective & Symptomatic & 0.15 per 1000 catheter-days & 18 \\
PICC & Prospective & Symptomatic & $18.7 \%$ & 26 \\
\hline CICC, cen & & \\
\hline
\end{tabular}

CICC, centrally inserted central catheter; CRT, catheter-related thrombosis; PICC, peripherally inserted central catheter.

\section{RISK FACTORS}

Several factors have been identified as potential predisposing factors for CRT (table 2). Some are related to patient-underlying pathology, others depend on the different CVC devices and techniques used. A summary of the risk factors that have been found associated with CRT is provided.

Interestingly, CVC-related thrombotic events tend to be more common during the first months after implantation. ${ }^{891416}$

\begin{tabular}{|c|c|c|c|}
\hline Risk factor & OR & Study type & Reference \\
\hline \multirow[t]{4}{*}{ Left-side insertion } & 3.86 & Prospective & 30 \\
\hline & 6.01 & Prospective & 6 \\
\hline & 16.3 & Prospective & 9 \\
\hline & - & Retrospective & 23 \\
\hline Subclavian venipuncture & 2.16 & Meta-analysis & 28 \\
\hline Port versus PICC & 0.43 & Meta-analysis & 28 \\
\hline \multirow[t]{3}{*}{ PICC versus other CVCs } & 2.55 & Meta-analysis & 36 \\
\hline & & Prospective & 12 \\
\hline & & Retrospective & 21 \\
\hline \multirow[t]{3}{*}{ Catheter tip malposition } & 1.92 & Meta-analysis & 28 \\
\hline & 4.05 & Prospective & 6 \\
\hline & & Prospective & 10 \\
\hline More than one insertion attempt & 5.5 & Prospective & 38 \\
\hline Personal history of DVT & 2.03 & Meta-analysis & 28 \\
\hline $\mathrm{BMI}>25$ & 1.9 & Prospective & 42 \\
\hline Chest radiotherapy & 7.01 & Prospective & 6 \\
\hline Factor V Leiden & 4.6 & Meta-analysis & 46 \\
\hline G20210A prothrombin mutation & 4.9 & Meta-analysis & 46 \\
\hline
\end{tabular}

\section{FACTORS RELATING TO THE DEVICE AND}

\section{TECHNIQUE USED}

\section{Insertion site}

Some studies have reported that left-side catheter insertion confers a higher risk of CRT when compared with right-side insertion. However, this notion has been challenged by recent prospective randomised studies in which the central line implantation side was not a predictive factor for catheter-related thrombotic events. Another recent study, although retrospective, described a positive association between left-side insertion and CRT only within a small subgroup of patients with metastatic cancer. ${ }^{20} \mathrm{~A}$ meta-analysis assessing risk factors for CRT in patients with cancer resulted in left-side insertion not reaching statistical significance. $^{21}$

Moreover, subclavian venipuncture has been associated with higher CRT incidence; a meta-analysis concluded an elevated VTE risk with an OR of $2.16 .{ }^{21}$ Neither anatomical nor technical factors could explain this relation.

\section{TYPES OF CVC VENOUS ACCESS}

CVCs can be categorised as:

A. Temporary

- Central non-tunnelled catheter (NTC).

- PICC.

B. Semipermanent

- Tunnelled catheter.

C. Implantable central venous ports (port)

A higher rate of complication in NTCs was reported more than PICCs and ports. According to the Centers for Disease Control and Prevention (CDC) guideline, PICCs had a lower rate of infection than NTCs, and totally implantable catheter had the lowest risk of catheter-related bloodstream infection (CRBSI). NTCs account for the majority of CRBSI. 


\section{PORTS}

In recent years, the use of ports has increased considerably. Implantable ports improve patient self-image, have no need for local catheter-site care, but are more expensive and require surgery for catheter removal. They have been reported to have significantly lower infection rates compared with other central venous access devices. A meta-analysis concluded implantable ports feature a lower risk of developing CRT when compared with PICCs, with an OR of $0.43 .^{21}$

While ports seem to be safer than other CVCs with CRT, they still confer a high VTE risk. Not all ports are equally safe; another relevant factor may be port placement. Despite the popularity of arm port placement due to patient comfort, a recent retrospective study suggests that the risk of CRT may be increased in patients with arm ports as opposed to chest ports; (9.5\%) of 147 patients with cancer with arm ports experienced UEDVT compared with only $3(2.0 \%)$ of 150 subjects with chest ports. ${ }^{22}$

The incidence increases when the catheter takes up more than $50 \%$ of the vessel lumen and particularly because the stress applied with everyday use when the port pocket is in the forearm and the vascular catheter crosses the elbow joint. Ports have fewer complications and feature a higher quality of life and patient satisfaction than PICCs and non-tunnelled CVCs (NTCs). ${ }^{23}$

A few studies showed that silicone ports would be more appropriate for patients who carry these types of ports than polyurethane because they reduce the number of complications caused by thrombosis; silicone ports produce numerous mechanical problems which need the removal of the ports and catheters quickly and do not appear in polyurethane catheters. Despite this, these types of devices are increasingly used in oncology patients, improving their quality of life. $^{24}$

\section{PERIPHERALLY INSERTED CENTRAL CATHETERS}

PICCs have been commonly placed in patients for anticancer therapies. They offer many advantages in avoiding mechanical complications such as pneumothorax and haemorrhage secondary to puncture. PICCs are non-tunnelled, central catheters inserted through a peripheral vein of the arm; they are 50-60 cm long and are usually made of silicone or second or thirdgeneration polyurethane. A potential problem is that they deteriorate patients' veins and can obstruct vessels in the long term that can delay chemotherapy cycles.

Their duration depends on the technique of insertion, stabilisation of the venous access device, patient compliance and nurse competence in the maintenance of the device. It is very useful in the haematology and oncology setting, especially for ambulatory therapy, because they can be safely used even in patients with extremely low platelet counts or at high risk of haemorrhage.
PICCs are currently being used extensively in clinical practice due to their multiple benefits, featuring a simple insertion procedure, cost-effectiveness and greater patient comfort. Nonetheless, these devices have disadvantages as well, one of those being an increased risk of CRT. A systematic review and metaanalysis reported an association with an OR of 2.55 between PICCs and VTE, the risk increases even more in the subgroup of patients with cancer with an OR of $6.67^{25}$

One study showed no significant difference in complication rates, including thrombotic events, in which PICC and non-tunnelled devices were compared. ${ }^{26}$ According to the CDC guideline, PICCs had a lower rate of infection than NTCs, and totally implantable catheters had the lowest risk of CRBSI. A systematic review found that although PICCs were associated with a lower risk of CRBSI than other CVCs in outpatients, hospitalised patients might be just as likely to experience CRBSI with PICCs as with other CVCs. ${ }^{25}$

\section{CVC TIP POSITION}

Studies are suggesting that the catheter tip position is a relevant factor concerning VTE risk. An initial distal tip placement in the superior vena cava and right atrial junction has been identified as a protective factor against CRT, probably due to the prevention of endothelial trauma and greater toxic substance dilution. Significantly higher VTE risk has been reported in patients with incorrect initial tip placement. ${ }^{6} 9$ According to a meta-analysis, placing the tip outside of this junction increases the risk of VTE with an OR of $1.92 .^{21}$

\section{INSERTION PROCEDURE}

The current standard of care for CVC insertion is an ultrasound-guided insertion, allowing a safe and easy procedure. However, this practice does not seem to have an impact on CRT.

Another study reported that more than one attempt at insertion was associated with a VTE OR of 5.5, while the precedent of a previous CVC also related positively, but with a slightly weaker OR of $3.8 .^{27}$ Furthermore, endothelial damage activating the coagulation cascade may be a reasonable pathophysiological explanation for this association.

\section{FACTORS RELATING TO THE UNDERLYING PATHOLOGY AND PATIENT HISTORY Infection}

Catheter infection is highly associated with intraluminal thrombosis and catheter-related venous thrombosis. An Australian study evaluated the rate of CVC-related complications showed that thrombosis occurred in $15.1 \%$ of all cases, exit-site infection in 1.9\% and CVC-related bloodstream infection in 7.5 per 1000 CVC-days. ${ }^{26}$ 
A case-control study reported an association between VTE and any recent infection in patients with cancer, with an OR of $1.7 .^{4}$

General guidelines for the prevention of intravascular catheter-related infections suggest the use of the subclavian vein, rather than a jugular or a femoral site, in adult patients to minimise infection risk for nontunnelled CVC placement. No recommendation can be made for a preferred site of insertion to minimise infection risk for a tunnelled CVC. Also, the use of a CVC with the minimum number of ports or lumens is essential for the management of the patient.

\section{MEDICAL HISTORY OF DVT}

Patients with a history of DVT should be considered at risk of future events. Previous DVT has been identified in a meta-analysis as an independent factor for CRT in patients with cancer. ${ }^{21}$

\section{BODY MASS INDEX}

Obesity, highly prevalent in contemporary medicine, is a known risk factor for VTE. The relationship between high body mass index (BMI) and CRT has been explored in some studies. A prospective study of patients with advanced cancer identified BMI greater than 25 as a statistically significant risk factor for PICC-related complications including CRT. ${ }^{28}$ However, other studies have failed to establish an association with statistical significance. ${ }^{622}$

\section{ADVANCED DISEASE}

It seems reasonable to expect patients with cancer in metastatic or other advanced stages to have a greater risk of VTE and there have been studies that report this association. ${ }^{42}$ The more aggressive underlying pathology is not the only explanation for this increased risk; thus, factors such as a higher likelihood of immobilisation must also be considered. Some studies have tried to establish an association between advanced cancer and CRT, but most have failed to reach statistical significance. ${ }^{781127}$ One prospective, randomised, double-blind, placebo-controlled study assessing the efficacy and safety of enoxaparin in the prevention of CRT reported a significantly higher CRT incidence in patients with metastatic disease, but only in the placebo arm. ${ }^{6}$

\section{CHEST RADIOTHERAPY}

Very few studies have assessed the association between chest radiotherapy and UEDVT; a randomised, doubleblind prospective study found a strong association with an OR of $7.01,^{6}$ while a retrospective study found no statistically significant results. ${ }^{22}$ The potential correlation between chest radiotherapy and CRT remains unclear until more studies can reproduce these results.

\section{THROMBOPHILIA}

Although patients with known congenital thrombophilias represent a small fraction of all patients with
Table 3 Khorana et a/ risk score for chemotherapy-related VTE risk prediction in patients with cancer ${ }^{31}$

\begin{tabular}{ll}
\hline Patient characteristic & Score \\
\hline $\begin{array}{l}\text { Site of cancer } \\
\text { Very high risk } \\
\text { (stomach, pancreas) }\end{array}$ & 2 \\
$\begin{array}{l}\text { High risk } \\
\text { (lung, lymphoma, gynaecological, genitourinary excluding } \\
\text { prostate) }\end{array}$ & 1 \\
$\begin{array}{l}\text { Prechemotherapy platelet count } \geq 350 \times 10^{9} / \mathrm{L} \\
\text { Prechemotherapy leucocyte count } \geq 11 \times 10^{9} / \mathrm{L}\end{array}$ & 1 \\
$\begin{array}{l}\text { Haemoglobin }<10 \mathrm{~g} / \mathrm{dL} \text { or use of erythropoiesis-stimulating } \\
\text { agents }\end{array}$ & 1 \\
\hline $\mathrm{BMI} \geq 35 \mathrm{~kg} / \mathrm{m}^{2}$ & 1 \\
\hline $\mathrm{BMI}$, body mass index; VTE, venous thromboembolism. & 1 \\
\hline
\end{tabular}

cancer requiring CVCs, the relationship of these diseases with CRT has been assessed. A meta-analysis concluded that factor V Leiden (FVL) and G20210A prothrombin mutation (PTM) thrombophilias in patients with cancer are associated with CRT, with pooled ORs of 4.6 and 4.9, respectively. Regarding impact, the reported attributable risk was $13.1 \%$ for FVL and $4.5 \%$ for PTM. ${ }^{29}$ Another article on the subject, a systematic review, concluded that FVL is associated with a greater incidence of CRT with a risk ratio of of 2.6-7.7, but found that the existing evidence is insufficient to conclude in respect of PTM and other rarer thrombophilias. ${ }^{30}$

\section{VTE RISK ASSESSMENT}

Khorana et al (table 3) elaborated a validated predictive score for chemotherapy-related thrombosis that considered cancer site, prechemotherapy platelet and leucocyte counts, haemoglobin levels and BMI. ${ }^{31}$

This score divides patients into three categories: low risk (score $=0$, confers $0.3 \%-0.8 \% 2.5$-month risk of VTE), intermediate risk (scores 1-2, 1.8\%-2\% VTE risk) and very high risk (scores 3 and above, 6.7\%7.1\% VTE risk). The model has limitations worth noting; since it considers prechemotherapy parameters, it can only be applied before the start of chemotherapy. In later studies the score has performed rather poorly, its accuracy being low in lung, colorectal and ovarian cancer. ${ }^{32}$ In the SAVE-ONCO Study, a doubleblind, randomised trial assessing the efficacy and safety of semuloparin for VTE prophylaxis in a group of 3212 patients with cancer undergoing chemotherapy, the score failed in predicting $70 \%$ of cancer-related thrombotic events observed. ${ }^{33}$

The development of new predictive models has been attempted. Of note is the COMPASS-CAT risk assessment model (table 4), a predictive score for VTE for patients with breast, colorectal, lung or ovarian cancer that considers the presence of a CVC as a risk factor for thrombosis. The variables taken into account are 
Table 4 COMPASS-CAT: a risk assessment model for cancerrelated VTE in breast, colorectal, lung and ovarian cancer $^{32}$

\begin{tabular}{ll}
\hline Patient characteristic & Score \\
\hline Cancer-related risk factors & 6 \\
\hline Antihormonal therapy or anthracycline & 4 \\
\hline Time since cancer diagnosis $\leq 6$ months & 3 \\
\hline CVC & 2 \\
\hline Advanced stage of cancer & 5 \\
Predisposing risk factors & \\
$\begin{array}{l}\text { Cardiovascular risk factors (at least } 2 \text { of the following } \\
\text { predictors: a personal history of peripheral artery disease, }\end{array}$ & \\
ischaemic stroke, coronary artery disease, hypertension, \\
hyperlipidaemia, diabetes, obesity) \\
$\begin{array}{l}\text { Recent hospitalisation for acute medical illness } \\
\text { Personal history of } V T E\end{array}$ \\
$\begin{array}{l}\text { Biomarkers } \\
\text { Platelet count } \geq 350 \times 10^{9} / L\end{array}$ \\
\hline
\end{tabular}

CVC, central venous catheter; VTE, venous thromboembolism.

classified as cancer-related risk factors, predisposing risk factors and laboratory biomarkers. ${ }^{32}$

Scores greater than or equal to 7 are considered at high risk for VTE, with a $13 \%$ risk of developing a thrombotic event. This is a recent study from 2017 that requires external validation. It requires greater diffusion and usage to compare the results of the original study with the general population. Therefore, due to the lack of a single-validated score or predictive model applicable to all types of cancer, the patient's risk of developing thrombotic events must be assessed on a case-by-case basis.

\section{OTHER RISKS OF CVCS IN PATIENTS WITH CANCER}

The NTCs in the internal jugular vein or subclavian vein cost less than other CVCs and are used when the chemotherapy process is short. Complications of NTCs were reported more than PICCs and ports.

Additionally, the use of venous ultrasound guidance has increased during venous puncture making CVC placement safer in patients with profound thrombocytopenia. Regarding risk associated with vascular access, the Consensus Guidelines for Periprocedural Management of Coagulation Status and Hemostasis Risk in Percutaneous Image-guided Interventions recommended the transfusion of platelets for any platelet count of less than $50 \times 10^{9} / \mathrm{L} .^{34}$

The Dutch guideline (recommends increasing the trigger to $20 \times 10^{9} / \mathrm{L}$ in case of fever and to $50 \times 10^{9} / \mathrm{L}$ for the use of anticoagulation. The British Committee for Standards in Hematology, the American Society of Clinical Oncology (ASCO) and the American Association of Blood Banks recommend likewise a more liberal transfusion policy in these conditions, but they do not specify triggers. ${ }^{35}$ The same heterogeneity in recommendations is seen in invasive procedures, such as lumbar punctures and insertion and removal of CVCs. Therefore, the decision to transfuse is based on the opinion of the treating physician and may differ significantly.

Prior to the removal of a CVC, $29 \%$ used a trigger of $10 \times 10^{9} / \mathrm{L}, 12 \%$ of $20 \times 10^{9} / \mathrm{L}$ and $59 \%$ used triggers between $30 \times 10^{9} / \mathrm{L}$ and $100 \times 10^{9} / \mathrm{L}$. The guideline advises maintaining the trigger of $10 \times 10^{9} / \mathrm{L} .{ }^{35}$

A prospective study that assessed haemorrhagic complication in 143 PICC implantations in 101 patients with thrombocytopenic cancer showed that $65 \%$ of the procedures were performed with a platelet count $20-50 \times 10^{9} / \mathrm{L}$ and $35 \%$ had lower than $20 \times 10^{9} /$ L. No major haemorrhage was observed. Minor oozing was observed in six implantations and mild haematoma in two for a total of eight minor haemorrhagic adverse events (5.5\%). In patients with a platelet count $<20 \times 10^{9} / \mathrm{L}, 1$ of $50(2 \%)$ had minor oozing and there were no cases of a minor haematoma. The authors suggest that PICCs in patients with a platelet count between $20 \times 10^{9} / \mathrm{L}$ and $50 \times 10^{9} / \mathrm{L}$ and lower than $20 \times 10^{9} / \mathrm{L}$ are safe even without platelet transfusions, provided that the patient does not have an associated secondary haemostatic abnormality and did not receive drugs that may have affected haemostatic status. ${ }^{36}$

Unfortunately, $30 \%$ of these devices fail before completion of treatment from complications with the highest prevalence in patients with cancer. Also, in a population with advanced cancers and receiving concurrent chemoradiotherapy, $15.1 \%$ exhibited PICC-related infectious complications, and 11.4\% developed symptomatic PICC-related thrombosis. ${ }^{28}$

\section{CRT AND THROMBOPROPHYLAXIS}

Due to their greater risk of VTE, it is not uncommon for these patients to receive thromboprophylaxis. With that said, in the right patient and setting, thromboprophylaxis is very useful for the prevention of thrombosis, but the efficacy of thromboprophylaxis for CRT is questionable. A randomised, double-blind, placebocontrolled trial assessing the efficacy of enoxaparin for CRT prevention resulted in no difference in the rates of CRT between the LMWH and placebo groups. ${ }^{37}$ A similar study that evaluated warfarin instead of LMWH also found similar rates of CRT in the warfarin and placebo groups, although in this study it may be attributable to the low incidence of thrombotic events reported. ${ }^{38} \mathrm{~A}$ meta-analysis assessing potential risk factors for CRT in patients with cancer found no statistically significant differences in CRT incidence between those that received thromboprophylaxis and those who did not. ${ }^{21}$

\section{TREATMENT OF CATHETER-ASSOCIATED DVT}

Manifestations include failure to draw blood from the port or failure of infusion. Thrombolytic therapy has been described to reverse these complications. All these complications may delay the chemotherapy course. Management of catheter-associated 


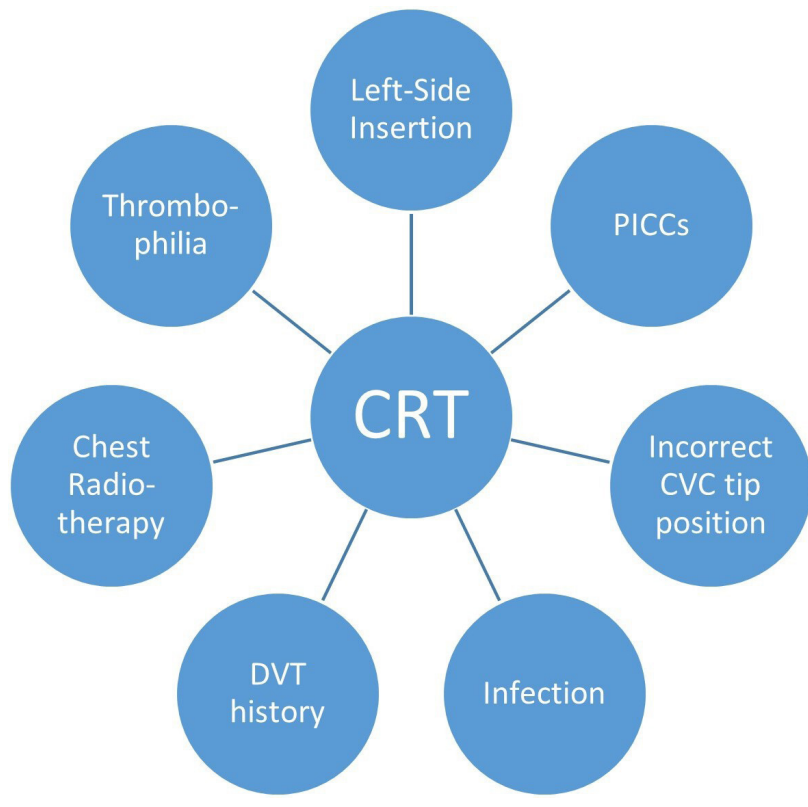

Figure 1 Risk factors featuring the strongest and/or most consistent association with CRT in the context of malignancy. CRT, catheter-related thrombosis; CVC, central venous catheter; DVT, deep vein thrombosis; PICCs, peripherally inserted central catheters.

thrombosis has not been well studied in patients with cancer. A prospective study looking at the use of anticoagulation for CRT in patients with cancer found no recurrent VTE and three cases of major bleeding in 74 patients with cancer who were treated with 5-7 days of LMWH followed by warfarin for 3 months. After 3 months, $57 \%$ of patients had functional catheters, and the remaining patients had their catheters removed for reasons other than progressive thrombosis or device failure. ${ }^{39}$ Catheter-associated thrombosis is thought to be associated with a low risk of recurrent VTE and post-thrombotic syndrome. Thus, venous catheters should be removed only in select situations, such as when the catheter is defective or no longer needed. Although malignancy is a risk factor for CRT, the main reason and determinant for CRT is the placement of the catheter tip high in the superior vena cava. Placement of the catheter tip high in the superior vena cava results in a higher incidence of thrombosis than when the catheter tip is placed low in the superior vena cava.

The International Society on Thrombosis and Hemostasis guidelines currently recommend that patients with cancer with catheter-associated thrombosis should be treated with therapeutic anticoagulation for 3 months. ${ }^{40}$ In patients with cancer with catheter-associated thrombosis, although LMWH is the suggested agent, vitamin $\mathrm{K}$ antagonists (VKAs) can also be used given the lack of direct comparative studies. Furthermore, CVCs can be kept in place if they are functional, well positioned and not infected. Currently, there is no standardised anticoagulation duration, whether the catheter is kept or removed.

The European Society for Medical Oncology recommends anticoagulation with LMWH, VKAs or LMWH followed by VKAs for 3-6months. If CVC is no longer needed or long-term anticoagulation is contraindicated, a short course of $3-5$ days is advised. CVC removal is only required in cases of concomitant DVT, sepsis or a non-functioning line. ${ }^{41}$

The National Comprehensive Cancer Network $(\mathrm{NCCN})$ guidelines for VTE in patients with cancer

\section{CRT - Treatment}

\section{Anticoagulation}

\section{-Who to treat:}

- Anticoagulation is beneficial in CRT but must be balanced with hemorrhagic risks

-What to treat with:

- Choice of agent is unclear: LMWH, VKA and NOACs may be effective

- LMWH monotherapy or LMWH bridged to warfarin are most commonly used

-Treatment duration:

- Unclear; ISTH guidelines suggest a period of 3 months

\section{Cathether removal}

\section{-Should be considered if:}

-Associated infection present

- Defective/dysfunctional line

- Line no longer necessary

Figure 2 Treatment strategies for CRT in the context of malignancy. CRT, catheter-related thrombosis; ISTH, International Society on Thrombosis and Hemostasis; LMWH, low-molecular-weight heparin; NOACs, novel oral anticoagulants; VKA, vitamin K antagonist. 
recommend anticoagulation for at least 3 months or for as long as the catheter is maintained. There are no specific drug recommendations from the NCCN. The CVC should be removed in case of persistent thrombosis symptoms, infection, dysfunctional line or when the line is no longer needed.

Generally, treatments for these patients may include LMWH bridged to warfarin or LMWH monotherapy, generally without line removal unless it is no longer required, it is defective or infection has been detected.

A retrospective analysis limited to 83 patients with CVC-associated DVT treated with rivaroxaban showed a line failure rate of $3.6 \%$ and a major bleeding rate of only $2.4 \%$ leading the authors to conclude that rivaroxaban was a safe and effective option. ${ }^{42}$

In a prospective study of rivaroxaban for CVCassociated UEDVT in patients with cancer, the authors demonstrated in 70 patients that preservation of line function was $100 \%$ at 12 weeks. The risk of recurrent VTE at 12 weeks was $1.43 \%$, with one episode of fatal $\mathrm{PE}$, and nine patients (12.9\%) experienced 11 total bleeding episodes. They concluded that rivaroxaban showed promise in treating CVC-UEDVT in patients with cancer, resulting in preserved line function. However, bleeding rates and a fatal PE on treatment are concerning safety outcomes necessitating further study before rivaroxaban can be recommended. ${ }^{43}$

Another study compared the efficacy and safety profile of rivaroxaban $(20 \mathrm{mg} /$ day $)$ with LMWH and VKAs in the treatment of PICC-associated UEDVT. Rivaroxaban led to faster resolution of PICC-associated UEDVT than LMWH/VKAs without any increase in bleeding. ${ }^{44}$

A recent Cochrane Database Systematic Review was published that studied the efficacy and safety of anticoagulation for thromboprophylaxis in people with cancer with a CVC. The evidence was not conclusive for the effect of LMWH compared with no LMWH on mortality, the effect of VKA compared with no VKA on mortality and CRT, and the effect of LMWH compared with VKA on mortality and CRT. They found moderate-certainty evidence that LMWH reduced CRT compared with no LMWH. In considering anticoagulation in patients with cancer with a CVC, the practitioner should assess the potential haemorrhagic complications associated with anticoagulants. ${ }^{45}$

Although most guidelines recommend anticoagulation for the treatment of CRT, the management of CRT with catheter removal alone may be considered for patients with a high risk of bleeding. A recent retrospective review comparing PICC removal alone versus PICC removal plus anticoagulation concluded that catheter removal alone results in significantly less major bleeding events at the expense of a small but significant increase in secondary VTE events and risk of VTE progression. ${ }^{46}$ An older retrospective study compared different treatment modalities for CRT: anticoagulation alone, CVC removal and anticoagulation,
CVC removal alone and CVC replacement. CRT replacement was the only modality that did not result in a $100 \%$ symptom resolution rate, with 4 out of 12 patients presenting persistent symptoms related to thrombosis. ${ }^{47}$ If no anticoagulation is being considered, whether central vascular access will be further needed, should be acknowledged.

\section{TREATMENT OF INTRALUMINAL THROMBOSIS}

Intraluminal thrombotic complications constitute a drastically different scenario than the previously discussed extraluminal thrombosis, both in presentation and therapeutic approach. A common cause of CVC dysfunction, intraluminal thrombosis usually manifests as total occlusion with failure to infuse into the CVC and absence of blood return. Fibrin sheath formation is a closely related phenomenon that generates a partial ball-valve effect. Thrombotic occlusion is usually managed with the administration of a thrombolytic agent, after exclusion of other causes of CVC dysfunction. Thrombolysis is considered a safe and simple procedure capable of restoring the CVC patency and avoiding unnecessary line replacement. Several different thrombolytic agents have been studied, urokinase being the most widely used. A recent meta-analysis reviewed the existing literature on the interventions to obstructive events in longterm CVCs in patients with cancer; the most common agents used were urokinase and alteplase. The overall restoration frequency was $84 \%$; the drugs analysed, with their respective restoration frequencies, consisted of urokinase (84\%), alteplase (92\%) and tenecteplase $(84 \%) .^{48}$ The ASCO guidelines currently recommend the instillation of $2 \mathrm{mg}$ of tissue plasminogen activator for the management of catheter-related occlusions. ${ }^{49}$

\section{CONCLUSION}

CRT is a moderately common clinical scenario in the oncological patient bearing a CVC. Although some devices are associated with greater risk, these patients harbour the risk of thrombosis regardless of the type of device used. Several factors relating both to technical aspects, patient and pathological characteristics may predispose to its occurrence; but until more studies on the subject are executed, their role and impact will remain unclear. Figure 1 depicts the most important risk factors found in association with CRT.

\section{What this study adds}

Despite its frequency, the optimal management strategy when facing this complication is still undetermined. A summary of the possible treatment strategies is provided in figure 2. It is not clear whether thromboprophylaxis is effective and/or required for CRT prevention. The existing evidence seems to suggest the best alternative for treatment in most cases is anticoagulation without CVC removal as long as the line is functional and free from infection and the benefits of anticoagulation outweigh the risks. Regarding the 
choice of anticoagulant, most seem to favour LMWH but the evidence about which agent to use is inconclusive. More research on the efficacy and safety of LMWH, VKA and direct oral anticoagulant, as well as studies comparing these drugs, is required in order to make an evidence-based decision.

Contributors AM-conception of the work, analysis and interpretation of data; manuscript writing and final approval of the manuscript. LB-analysis and interpretation of data; manuscript writing and final approval of the manuscript. MA-substantial contributions to the conception of the work, analysis and interpretation of data; manuscript writing and final approval of the manuscript. MK-editing, formatting and revising for clarification of conceptual work.

Funding The authors have not declared a specific grant for this research from any funding agency in the public, commercial or not-for-profit sectors.

Competing interests None declared.

Patient consent for publication Not required.

Provenance and peer review Not commissioned; externally peer reviewed.

Open access This is an open access article distributed in accordance with the Creative Commons Attribution Non Commercial (CC BY-NC 4.0) license, which permits others to distribute, remix, adapt, build upon this work noncommercially, and license their derivative works on different terms, provided the original work is properly cited, appropriate credit is given, any changes made indicated, and the use is noncommercial. See: http://creativecommons.org/licenses/by-nc/4. $0 \%$

\section{ORCID iD}

Matthew Kinzie http://orcid.org/0000-0001-6496-4093

\section{REFERENCES}

1 Bleker SM, van Es N, van Gils L, et al. Clinical course of upper extremity deep vein thrombosis in patients with or without cancer: a systematic review. Thromb Res 2016;140:S81-8.

2 Sørensen HT, Mellemkjær L, Olsen JH, et al. Prognosis of cancers associated with venous thromboembolism. N Engl J Med Overseas Ed 2000;343:1846-50.

3 Galanaud J-P, Blaise S, Sevestre M-A, et al. Long-Term outcomes of isolated superficial vein thrombosis in patients with active cancer. Thromb Res 2018;171:179-86.

4 Ashrani AA, Gullerud RE, Petterson TM, et al. Risk factors for incident venous thromboembolism in active cancer patients: a population based case-control study. Thromb Res 2016;139:29-37.

5 Kuter DJ. Thrombotic complications of central venous catheters in cancer patients. Oncologist 2004;9:207-16.

6 Verso M, Agnelli G, Kamphuisen PW, et al. Risk factors for upper limb deep vein thrombosis associated with the use of central vein catheter in cancer patients. Intern Emerg Med 2008;3:117-22.

7 De Cicco M, Matovic M, Balestreri L, et al. Central venous thrombosis: an early and frequent complication in cancer patients bearing long-term silastic catheter. A prospective study. Thromb Res 1997;86:101-13.

8 Boddi M, Villa G, Chiostri M, et al. Incidence of ultrasounddetected asymptomatic long-term central vein catheterrelated thrombosis and fibrin sheath in cancer patients. Eur J Haematol 2015;95:472-9.

9 Luciani A, Clement O, Halimi P, et al. Catheter-Related upper extremity deep venous thrombosis in cancer patients: a prospective study based on Doppler us. Radiology 2001;220:655-60.
10 Van Rooden CJ, Rosendaal FR, Barge RMY, et al. Central venous catheter related thrombosis in haematology patients and prediction of risk by screening with Doppler-ultrasound. Br J Haematol 2003;123:507-12.

11 Patel GS, Jain K, Kumar R, et al. Comparison of peripherally inserted central venous catheters (PICC) versus subcutaneously implanted port-chamber catheters by complication and cost for patients receiving chemotherapy for non-haematological malignancies. Support Care Cancer 2014;22:121-8.

12 Kim HJ, Yun J, Kim HJ, et al. Safety and effectiveness of central venous catheterization in patients with cancer: prospective observational study. J Korean Med Sci 2010;25:1748-53.

13 Cortelezzi A, Moia M, Falanga A, et al. Incidence of thrombotic complications in patients with haematological malignancies with central venous catheters: a prospective multicentre study. Br J Haematol 2005;129:811-7.

14 Napalkov P, Felici DM, Chu LK, et al. Incidence of catheterrelated complications in patients with central venous or hemodialysis catheters: a health care claims database analysis. BMC Cardiovasc Disord 2013;13:81.

15 Lipitz-Snyderman A, Ma Q, Pollack MF, et al. Complications associated with use of long-term central venous catheters among commercially insured women with breast cancer. JOP 2015;11:505-10.

$16 \mathrm{Vu} \mathrm{K}$, Luong NV, Hubbard J, et al. A retrospective study of venous thromboembolism in acute leukemia patients treated at the University of Texas MD Anderson Cancer Center. Cancer Med 2015;4:27-35.

17 Masci G, Magagnoli M, Pedicini V, et al. Long-Term, tunneled, noncuffed central venous catheter in cancer patients (Vygon): safety, efficacy, and complications. Support Care Cancer 2006;14:1141-6.

18 Campagna S, Gonella S, Berchialla P, et al. Can peripherally inserted central catheters be safely placed in patients with cancer receiving chemotherapy? A retrospective study of almost 400,000 Catheter-Days. Oncologist 2019;24:e953-9.

19 Schwarz RE, Coit DG, Groeger JS. Transcutaneously tunneled central venous lines in cancer patients: an analysis of devicerelated morbidity factors based on prospective data collection. Ann Surg Oncol 2000;7:441-9.

20 El-Balat A, Schmeil I, Karn T, et al. Catheter-Related complications of subcutaneous implantable venous access devices in breast cancer patients. In Vivo 2018;32:1275-81.

21 Saber W, Moua T, Williams EC, et al. Risk factors for catheterrelated thrombosis (crt) in cancer patients: a patient-level data (IPD) meta-analysis of clinical trials and prospective studies. $J$ Thromb Haemost 2011;9:312-9.

22 Tippit D, Siegel E, Ochoa D, et al. Upper-Extremity deep vein thrombosis in patients with breast cancer with chest versus arm central venous port catheters. Breast Cancer 2018;12:117822341877190.

23 Fang S, Yang J, Song L, et al. Comparison of three types of central venous catheters in patients with malignant tumor receiving chemotherapy. Patient Prefer Adherence 2017;11:1197-204.

24 Alfonso Alvarez-Rodríguez J, García-Suárez M, FernándezGarcía D, et al. Analysis of peripheral central venous access ports at the forearm: an observational study. Eur J Cancer Care 2018;27:e12929.

25 Chopra V, Anand S, Hickner A, et al. Risk of venous thromboembolism associated with peripherally inserted central catheters: a systematic review and meta-analysis. The Lancet 2013;382:311-25.

26 Worth LJ, Seymour JF, Slavin MA. Infective and thrombotic complications of central venous catheters in patients with hematological malignancy: prospective evaluation of nontunneled devices. Support Care Cancer 2009;17:811-8. 
27 Lee AYY, Levine MN, Butler G, et al. Incidence, risk factors, and outcomes of catheter-related thrombosis in adult patients with cancer. J Clin Oncol 2006;24:1404-8.

28 Xie J, Xu L, Xu X, et al. Complications of peripherally inserted central catheters in advanced cancer patients undergoing combined radiotherapy and chemotherapy. J Clin Nurs 2017;26:4726-33.

29 Dentali F, Gianni M, Agnelli G, et al. Association between inherited thrombophilic abnormalities and central venous catheter thrombosis in patients with cancer: a meta-analysis. $J$ Thromb Haemost 2008;6:70-5.

30 Boersma RS, Hamulyak K, Cate HT, et al. Congenital thrombophilia and central venous catheter-related thrombosis in patients with cancer. Clin Appl Thromb Hemost 2010;16:643-9.

31 Khorana AA, Kuderer NM, Culakova E, et al. Development and validation of a predictive model for chemotherapyassociated thrombosis. Blood 2008;111:4902-7.

32 Gerotziafas GT, Taher A, Abdel-Razeq H, et al. A predictive score for thrombosis associated with breast, colorectal, lung, or ovarian cancer: the prospective COMPASS-Cancer-Associated thrombosis study. Oncologist 2017;22:1222-31.

33 Agnelli G, George DJ, Kakkar AK, et al. Semuloparin for thromboprophylaxis in patients receiving chemotherapy for cancer. N Engl J Med 2012;366:601-9.

34 Malloy PC, Grassi CJ, Kundu S, et al. Consensus guidelines for periprocedural management of coagulation status and hemostasis risk in percutaneous image-guided interventions. $J$ Vasc Interv Radiol 2009;20:S240-9.

35 Kaufman RM, Djulbegovic B, Gernsheimer T, et al. Platelet transfusion: a clinical practice guideline from the AABB. Ann Intern Med 2015;162:205.

36 Potet J, Thome A, Curis E, et al. Peripherally inserted central catheter placement in cancer patients with profound thrombocytopaenia: a prospective analysis. Eur Radiol 2013;23:2042-8.

37 Verso M, Agnelli G, Bertoglio S, et al. Enoxaparin for the prevention of venous thromboembolism associated with central vein catheter: a double-blind, placebo-controlled, randomized study in cancer patients. JCO 2005;23:4057-62.

38 Couban S, Goodyear M, Burnell M, et al. Randomized placebo-controlled study of low-dose warfarin for the prevention of central venous Catheter-Associated thrombosis in patients with cancer. JCO 2005;23:4063-9.

39 Kovacs MJ, Kahn SR, Rodger M, et al. A pilot study of central venous catheter survival in cancer patients using low- molecular-weight heparin (dalteparin) and warfarin without catheter removal for the treatment of upper extremity deep vein thrombosis (the catheter study). J Thromb Haemost 2007;5:1650-3.

40 Debourdeau P, Farge D, Beckers M, et al. International clinical practice guidelines for the treatment and prophylaxis of thrombosis associated with central venous catheters in patients with cancer. J Thromb Haemost 2013;11:71-80.

41 Sousa B, Furlanetto J, Hutka M, et al. Central venous access in oncology: ESMO clinical practice guidelines. Ann Oncol 2015;26 Suppl 5:v152-68.

42 Laube ES, Mantha S, Samedy P, et al. Treatment of central venous catheter-associated deep venous thrombosis in cancer patients with rivaroxaban. Am J Hematol 2017;92:E9-10.

43 Davies GA, Lazo-Langner A, Gandara E, et al. A prospective study of rivaroxaban for central venous catheter associated upper extremity deep vein thrombosis in cancer patients (catheter 2). Thromb Res 2018;162:88-92.

44 Fan F, Zou Y, Zhang S, et al. Rivaroxaban in the treatment of PICC-associated upper extremity venous thrombosis. Clin Ther 2017;39:1882-8.

45 Kahale LA, Tsolakian IG, Hakoum MB, et al. Anticoagulation for people with cancer and central venous catheters. Cochrane Database Syst Rev 2018;112.

46 Shatzel JJ, Mart D, Bien JY, et al. The efficacy and safety of a catheter removal only strategy for the treatment of PICC line thrombosis versus standard of care anticoagulation: a retrospective review. J Thromb Thrombolysis $2019 ; 47: 585-9$.

47 Frank DAet al. The treatment and outcome of cancer patients with thromboses on central venous catheters. J Thromb Thrombolysis 2000;10:271-5.

48 da Costa ACC, Ribeiro JM, Vasques CI, et al. Interventions to obstructive long-term central venous catheter in cancer patients: a meta-analysis. Support Care Cancer 2019;27:407421.

49 Schiffer CA, Mangu PB, Wade JC, et al. Central venous catheter care for the patient with cancer: American Society of clinical oncology clinical practice guideline. JCO 2013;31:1357-70.

50 Haggstrom L, Parmar G, Brungs D. Central venous catheter thrombosis in cancer: a multi-centre retrospective study investigating risk factors and contemporary trends in management. Clin Med Insights Oncol 2020;14:117955492095309. 